\title{
Balkanologie
}

Balkanologie Revue d'études pluridisciplinaires

Vol. VIII, $n^{\circ} 1 \mid 2004$

Volume VIII Numéro 1

\section{La justice internationale dans l'après-guerre}

La difficile évaluation des critères de justice

Post-war International Justice. The Laborious Evaluation of Justice Critions

\section{Isabelle Delpha}

\section{(2) OpenEdition}

Journals

Édition électronique

URL : http://journals.openedition.org/balkanologie/528

DOI : $10.4000 /$ balkanologie.528

ISSN : 1965-0582

Éditeur

Association française d'études sur les Balkans (Afebalk)

Édition imprimée

Date de publication : 1 juin 2004

ISSN : 1279-7952

\section{Référence électronique}

Isabelle Delpha, "La justice internationale dans l'après-guerre », Balkanologie [En ligne], Vol. VIII, n 1 I 2004, mis en ligne le 21 janvier 2010, consulté le 17 décembre 2020. URL : http://

journals.openedition.org/balkanologie/528 ; DOI : https://doi.org/10.4000/balkanologie.528

(c) Tous droits réservés 


\title{
LA JUSTICE INTERNATIONALE DANS L'APRÈS-GUERRE : LA DIFFICILE ÉVALUATION DES CRITĖRES DE JUSTICE
}

\author{
Isabelle Delpha*
}

L'idée qu'il n'y a " pas de paix sans justice " et que la justice pénale peut et doit contribuer à la réconciliation s'est imposée parmi les discours dominants concernant les processus de sortie de violence et d'après-guerre, notamment dans les Balkans ${ }^{1}$. Ce discours, tenu principalement par le Tribunal Pénal International pour l'ex-Yougoslavie (TPIY), les juristes internationaux et les associations de défense des droits de l'homme, donne comme mission au droit et à la justice pénale de contribuer à la " restauration et au maintien de la paix $\|^{2}$ et à " faire cesser les violations [du droit humanitaire] et à en réparer effectivement les effets $" 3$. Plus largement encore, cette justice dans les déclarations de ses promoteurs, tels que Madeleine Albright, est " essentielle pour adoucir l'amertume des familles de victimes, écarter des obstacles à la coopération entre les parties, établir un modèle de résolution des différences ethniques par

\footnotetext{
Maitre de conférences au département de philosophie de l'université Paul Valéry-Montpellier III. (IDelpha@aol.com)

${ }^{1}$ Cet article émane non d'une spécialiste des Balkans mais d'une philosophe, qui dans le cadre d'un travail théorique sur la justice internationale mène un travail empirique sur la réception et les effets de cette justice dans les Balkans, et principalement en Bosnie-Herzégovine (B.-H.) (programme financé par le ministère de la Recherche dans le cadre d'une ACI-jeunes chercheurs). Cette enquête de terrain, toujours en cours, a èté menée à La Haye en septembre 2002 et en B.-H. d'avril à juillet 2002 principalement à Sarajevo et dans sa banlieue, ainsi qu'à Banja Luka et occasionnellement à Klujč et Sanski Most, auprès d'associations de victimes (associations de familles de disparus, associations de victimes civiles, association d'anciens détenus des camps) ou d'associations de rẻfugiés et de "returneesn et des principaux interlocuteurs de cette justice internationale (" out reach program " du TPIY, Commission fédérale de recherche des disparus, ICMP) ainsi qu'avec de simples particuliers, réfugiés, " returnees ", témoins à La Haye, entre autres. Des associations analogues de disparus et de réfugiés ont été rencontrées à Belgrade en juin 2003 .
}

${ }^{2}$ Résolution du conseil de sécurité de l'ONU, S/ 25314, du 22 février 1993.

${ }^{3}$ Résolution 827 du 25 mai 1993. 
la force de la loi et non par la loi de la force ${ }^{4}$. Une telle mission de paix et de réconciliation serait accomplie par l'établissement de la vérité et l'assignation de responsabilités individuelles, évitant la stigmatisation de groupes entiers.

Les fins ainsi proposées à la justice pénale internationale réunissent des sources théoriques diverses, voire divergentes. L'idée kantienne de la paix internationale par le droit s'y enrichit d'une conception utilitariste qui juge de la fonction du droit et de celle de la peine par leur utilité sociale, en l'occurrence la dissuasion des crimes futurs et la réconciliation. Le TPI se trouverait ainsi à l'articulation d'une logique libérale de la règle de droit et de la responsabilité individuelle, d'un argumentaire de la paix (internationale) par la vérité, hérité de Nuremberg et de sa fonction pédagogique, et d'un argumentaire de la paix comme réconciliation, inspiré des commissions de vérité et réconciliation (CVR) dont le ressort est le pardon plutôt que les procédures judiciaires.

La diversité de ces sources théoriques, qu'il ne s'agit pas ici de discuter en tant que telles, éclaire partiellement l'une des difficultés majeures de l'évaluation des fins proposées à cette justice. Les déclarations de principe sur les vertus de la justice internationale sont rarement confrontées à la situation effective des pays concernés. Elles ont été acceptées comme une évidence parmi les militants des droits de l'homme, sans qu'elles ne soient guère ni confirmées, $\mathrm{ni}$ infirmées par des études empiriques sur les effets de ces tribunaux. Les effets de la justice internationale en termes de contribution à la paix et à la réconciliation, se trouvent donc stipulés plutôt qu'observés. Ils ont été supposés et transposés par analogie d'un cadre national à un cadre international, comme la fonction supposée de dissuasion, ou d'une expérience historique, notamment celle des procès de Nuremberg, à une autre. Ils ont aussi été extrapolés et importés à partir d'autres expériences de justice de transition dans les démocraties émergentes, comme les $\mathrm{CVR}^{5}$, en dépit de différences flagrantes de contextes et de procédures, ces commissions étant plus axées sur la vérité que sur la justice.

Inversement, les rares recherches (universitaires ou journalistiques) effectuées sur le terrain, en ex-Yougoslavie, constatant aisément le décalage entre ces déterminations normatives et leurs effets empiriques, concluent à une quasi absence d'effets de la justice dans un processus de reconstruction. On peut donc alternativement défendre que cette justice est faite au nom des victimes et participe d'un modèle de justice reconstructive du lien social ${ }^{6}$ ou objecter qu'elle a

\footnotetext{
4 Déclaration de Madeleine Albright au TPIY le 28 mai 1997, citée par Bass (Gary Jonathan), Stay the Hand of vengeance, Princeton : Princeton University Press, 2000, p. 284.

5 Voir Fletcher (Laurel), Weinstein (Harvey), "Violence and social repair : rethinking the contribution of justice to reconciliation ", Human Rights Quarterly, 24 (3), août 2002, pp. 593 et sq. À bien des égards, il faudrait considérer qu'il s'agit d'une extrapolation à partir des effets supposés de ces CVR.
}

${ }^{6}$ Garapon (Antoine), Des crimes que l'on ne peut ni punir ni pardonner, Paris : Odile Jacob, 2002, chapitre 8. 
été faite davantage au bénéfice des juristes internationaux que des victimes ${ }^{7}$ : les données et les analyses manquent pour évaluer la justesse de ces énoncés.

Toutefois, conclure à une absence d'effets de cette justice internationale dans un processus d'après-guerre serait aussi hâtif que les déclarations de principe sur son efficacité révolutionnaire. Le décalage entre les attentes et les événements n'est pas le signe d'un divorce et d'un discrédit tels que ceux qui, au Rwanda, affectent le TPIR, dont la rupture est consommée avec le gouvernement et les associations de victimes. Il n'y a ni adéquation ni séparation entre les fins du TPIY et celles des acteurs locaux. La place du TPIY dans l'aprèsguerre engage à un double travail : d'une part, empirique, de description, d'autre part, conceptuel, de détermination des critères d'évaluation de cette justice. Comme l'a déjà souligné Gary J. Bass dans son ouvrage sur la politique des tribunaux pour crimes de guerre au XIX ${ }^{\mathrm{e}}$ et $\mathrm{XX}^{\mathrm{e}}$ siècle, l'évolution des pays d'exYougoslavie $^{8}$, en relation avec la justice internationale, déjoue les prédictions des " réalistes " pour qui cette justice ne peut qu'être un obstacle à la paix et à un processus politique de normalisation. Elle déjoue également celle des promoteurs de cette justice, appelés par commodité libéraux ou « idéalistes " (par opposition aux " réalistes "), qui voient en elle la voie royale vers la paix et la réconciliation. Pour rendre compte d'un tel décalage entre les attentes et la réalité, il convient de redéfinir les critères appliqués à cette justice, aucun n'étant directement opératoire ni non plus impertinent, c'est-à-dire : la dissuasion de crimes futurs, la contribution à la paix et à la réconciliation, l'établissement des faits et de la vérité et la lutte contre le négationnisme, le combat contre la culpabilité collective par l'individualisation de la responsabilité.

\section{DES PRÉVISIONS EN PORTE-À-FAUX}

À ce jour, les analyses de Bass restent dans leurs grandes lignes valides. L'évolution politique des pays des Balkans ne confirme pas les prévisions alarmistes des réalistes. Selon eux, l'arrestation de criminels présumés et le spectacle de tribunaux et de procès imposés de l'extérieur peuvent entraîner une violente réaction nationaliste ou empêcher des compromis nécessaires à la paix. Or, l'inculpation de Karadžić a plutôt aidé Holbrooke à le marginaliser dans les négociations de paix alors que l'inculpation de Milošević pour le Kosovo n'a pas empêché l'OTAN de négocier un cessez-le-feu avec son régime. Plus généralement, jusqu'à maintenant, l'arrestation de criminels de guerre présumés a pu entraîner des manifestations, parfois violentes, comme en

7 Comme le suggère Neuffer (Elizabeth), The Key to my Neighbourgh House, Seeking Justice in Bosnia and Rwanda, New York : Picador, 2001, p. 391.

${ }^{8}$ Bass (Gary Jonathan), op.cit., voir l'épilogue. 
Serbie, mais pas de reprise du conflit, alors que des incidents bien plus sérieux ont pu se produire lors de rencontres sportives ou de la construction d'édifices religieux comme à Banja Luka ${ }^{9}$. Si l'on prend le critère des résultats électoraux, en Bosnie-Herzégovine (B.-H.), ces arrestations n'ont pas entraîné de poussée nationaliste. Au pire, elles ont laissé subsister l'emprise de partis impliqués dans le nettoyage ethnique, et déjà bien implantés. Au mieux, ces arrestations ont été suivies d'une victoire électorale de partis plus modérés comme à Prijedor. Plus largement, on ne constate pas de corrélation entre poussée électorale des partis nationalistes et prise de position sur le TPIY : il est indéniable que l'opposition au TPIY a servi l'argumentaire nationaliste en Serbie aux élections de décembre 2003 mais un retour au pouvoir des partis nationalistes s'est également produit avec celui du SDA en B.-H. en octobre 2002 et du HDZ en Croatie en novembre 2003 , sans qu'il y ait de corrélation entre défaite ou victoire électorale et prise de position ou degré de coopération avec le TPIY.

De même, les inculpations et les procès de La Haye ne peuvent être considérés comme un obstacle à la réconciliation. D'une part, les partis les plus opposés à une coopération avec le TPIY le sont aussi à une réconciliation alors que les échanges d'excuses et les demandes de pardon entre la Croatie et la Serbie ou de la part de la Serbie à la Bosnie ont émané d'hommes et de partis politiques les plus favorables à une coopération avec le TPIY ${ }^{10}$. Une remarque analogue peut être faite si l'on prend le critère de l'adhésion à l'Union européenne comme gage de stabilité et d'adhésion à des principes de paix. Les mouvements et partis politiques les plus favorables à une intégration européenne le sont également à une coopération avec le TPIY, les moins favorables finissent par accepter cette coopération si une telle intégration est pour eux une priorité. Lorsque le refus de coopération avec La Haye s'est révélé un obstacle direct à une candidature de la Croatie à l'Europe, le parti HDZ qui s'était violemment opposé à une telle coopération, a, en la personne d'Ivo Sanader, changé de position en favorisant le départ à La Haye de plusieurs inculpés Croates et Bosniaques ${ }^{11}$ croates d'Herzégovine, en avril $2004^{12}$.

9 Le 7 Mai 2001 lors de la pose de la première pierre pour la reconstruction de la mosquée Ferhadija, de violentes émeutes ont eu lieu où des nationalistes serbes ont pris pour cible des Bosniens et des représentants internationaux.

${ }^{10}$ Le 10 septembre 2003 , le président de Croatie, Stipe Mesić, et son homologue de Serbie-Monténégro, Marović, ont présenté des excuses mutuelles pour le mal commis par les citoyens de leur deux pays. Marović a également présenté des excuses à la B.-H. le 13 octobre 2003.

11 J'emploie le terme " Bosniaque " pour l'ensemble des citoyens de Bosnie-Herzégovine en distinguant éventuellement entre Croates, Serbes et Bosniens, pour indiquer la nationalité et non seulement la citoyenneté, le terme " Bosnien " référant plus spécifiquement aux Musulmans bosniaques.

${ }^{12}$ Le 12 Mars 2004, les généraux croates Čermak et Markac se sont présentés devant la cour de La Haye suite à leur inculpation pour l'opération « Tempête n. Le 5 avril Prlić, Stojić, Praljak, Petković, Corić et Pušić se sont tous rendus volontairement à La Haye pour des crimes commis contre les non-Croates de B.-H. 
Globalement, le TPIY n'empêche pas les différents partis politiques de poursuivre le programme qui est le leur. La référence au TPIY peut être intégrée dans leurs discours et stratégies pour alimenter l'argumentaire ultra-nationaliste du parti radical serbe de Šešelj et Nikolić ou a contrario l'argumentaire réformateur des partis libéraux, mais il ne le détermine pas. L'obligation imposée à la Croatie de réformer le droit des minorités ou de livrer des inculpés est respectée dans la mesure où l'Union européenne en fait une condition d'adhésion.

Les inculpations, les arrestations et les procès ne donnent pas pour autant raison aux libéraux selon lesquels un pays ne peut être stable si des criminels de guerre restent au pouvoir. Leur élimination ne suffisant pas à diminuer leur prestige aux yeux d'une population qui voyait en eux des héros nationaux, il faut qu'ils soient jugés dans des procès publics et équitables. Assurément, l'inculpation a correspondu à l'éviction de la scène politique de plusieurs prévenus, comme dans le cas de Stakić, ancien maire de Prijedor. Toutefois, ni les inculpations ni les procès ne suffisent à une telle éviction. Des inculpés toujours en fuite, comme Karadžić, Mladić ou Gotovina, peuvent garder leur statut de héros et leurs réseaux de pouvoir. Si les jugements peuvent correspondre à un affaiblissement d'influence politique pour Plavšić, Milošević et le parti socialiste de Serbie, cet affaiblissement avait en l'occurrence précédé les procès, et ne s'observe pas dans le cas du parti radical serbe de Šešelj.

De même, d'autres arguments des " idéalistes " en faveur de cette justice semblent ou bien directement réfutés, ou au moins ambigus dans leur prétendus effets.

L'idée que de tels tribunaux puissent avoir un effet dissuasif et empêcher des crimes futurs qui peut être invoquée pour l'opération Tempête ${ }^{13}$ est néanmoins manifestement battue en brèche par le massacre de Srebrenica et l'expulsion des Kosovars après l'instauration du tribunal ${ }^{14}$. Toutefois une telle prétention dissuasive étonne autant que ce type de réfutation ${ }^{15}$. Tous deux reposent sur l'extrapolation directe vers un espace international d'une conception utilitariste de la peine comme dissuasion qui a d'autant moins de force que la sanction est d'autant moins probable. Dans un espace international où l'impunité est la règle, la probabilité d'échapper à toute sanction est d'autant plus grande que les inculpations des TPI restent sporadiques en regard du

\footnotetext{
${ }^{13}$ D'autres exemples d'effets dissuasifs sont donnés par Bass (Gary Jonathan), op.cit., p. 294.
}

14 La crédibilité du tribunal pourrait aussi bien se mesurer aux efforts mis dans la dissimulation des traces et des preuves du crime, dans le déplacement des charniers et dans la destruction des corps, qui ont pris une grande ampleur après Srebrenica et le Kosovo.

15 L'idée que ce pouvoir dissuasif est mis à mal par les massacres de Srebrenica et du Kosovo est plus récemment reprise dans Snyder (Jack), Vinjamuri (Leslie), "Trials and errors ", International Security, 28 (23), 2004. 
nombre de criminels de guerre présumés. C'est seulement si le jugement des violations graves du droit humanitaire international devenait aussi systématique que celui des crimes de droit commun que l'analogie qui sous-tend l'argument serait valide.

En revanche, il est dommageable pour une compréhension des effets et de la réception du TPIY qu'un discours général sur les vertus de la lutte contre l'impunité contraste avec la faiblesse et le caractère tardif des réflexions sur les peines du TPIY ${ }^{16}$. Or, les arrestations et les peines sont ce par quoi le TPIY mord le plus directement sur l'opinion en Bosnie, par exemple. Des peines jugées peu cohérentes entre elles et incompréhensibles tendent à éloigner les Bosniens ${ }^{17}$ du TPIY. Des peines jugées trop basses pour Plavšić (11 ans) leur apparaissent comme une négation de l'ampleur des crimes entrant en contradiction avec une fonction de reconnaissance de la vérité que les jugements et plaidoyers de culpabilité visaient à assurer.

Le critère de prévention de la vengeance ${ }^{18}$ semble aussi peu convaincant. Selon cet argument, les procès sont préférables à des actes de vengeance, suscités par les violences de guerre ou par l'exécution sommaire des vaincus telle que Staline la souhaitait à la place du procès de Nuremberg ou que Tito l'a pratiquée à Bleiburg. Cependant, pour l'heure, on ne peut guère établir de corrélation entre adhésion au TPIY et diminution des vengeances. Selon un sondage réalisé l'hiver 2002 (par le South East Europe Democracy Support Network), le tribunal recevait la confiance de 3,6\% des habitants de RS, de $6 \%$ en Serbie, de $50,5 \%$ d'habitants de la fédération de $\mathrm{BH}$ et de $83,3 \%$ au Kosovo, où a lieu un grand nombre d'actes de vengeance contre les Serbes.

Plus convaincant est le critère d'établissement des faits et de la vérité. La visée de la justice est double, établir la vérité et reconnaître ou faire reconnaître ce qui s'est passé pour lutter contre le déni et le négationnisme. Étant donné la masse de documentation recueillie par le TPIY, le nombre de témoins interrogés et contre interrogés, le travail d'investigation et d'exhumation de charniers qui ne l'aurait pas été sans celui-ci, cette tâche est la plus clairement menée à bien. Avec des nuances toutefois. Même sans tenir compte des rap-

\footnotetext{
${ }^{16}$ Pour le manque de cohérence dans les peines du tribunal, voir notamment " Sentencing guidelines urged ", Tribunal update, (347), 8 mars 2004. Parmi les rares réflexions sur les peines en droit pénal international, voir Poncela (Pierrette), " Mesure et motivation de la peine dans les jugements du TPIY n, in Henzelin (Marc), Roth (Robert), éds., Le droit pénal à l'épreuve de l'internationalisation, Paris / Bruxelles / Genève : L.G.D.J. / Bruylant / Georg, 2002, et la thèse en cours de Liwerant (0. Sara), Le droit pénal face à la logique meurtrière des crimes contre l'humanité et des génocides. Regard criminologique et anthropologique sur les impensés du Droit, Nanterre.

${ }^{17}$ Je fais ici état des réactions publiées dans la presse bosniaque émanant d'associations de victimes bosniennes, d'organisations bosniennes comme la Commission Fédérale pour la recherche des disparus, lors du jugement Plavšić et de plusieurs plaidoyers de culpabilité, notamment pour Srebrenica, qui ont suivi le jugement Plavšić.
}

${ }^{18}$ Ce critère d'effectivité des TPI est proposé par Bass (Gary Jonathan), op.cit., p. 304 et sq. 
ports délicats entre vérité judiciaire et vérité historique, entre travail du juge et de l'historien, ce type de justice n'établit pas les faits, mais seulement certains faits, exhume certains charniers, sélectionne certains témoins en fonction des actes d'accusation. Et même dans ce cas, la distance est grande entre l'établissement de ces faits par un tribunal d'une part et leur connaissance et reconnaissance d'autre part. D'une part, les jugements du TPIY, notamment les récents plaidoyers de culpabilité de plusieurs Serbes bosniaques, sont peu relayés et commentés par les média locaux, notamment en Republika Srpska (RS), donc peu accessibles. Ce n'est qu'en 1999 que la présidente du TPIY, Gabrielle Mac Donald, préoccupée par cette distance entre le TPIY et les pays concernés, a proposé l'instauration de "out reach programs " de communication à destination des populations intéressées ${ }^{19}$. Et ce n'est qu'en février 2002 qu'un correspondant permanent pour un journal bosniaque, Dani en l'occurrence, est arrivé à La Haye. D'autre part, l'idée que l'établissement des faits suffirait à leur reconnaissance repose sur une conception de la vérité comme index sui et falsum qui ne tient compte ni des divergences entre vérité du témoin et vérité du juge ou de l'historien ni des conditions sociales d'acceptation de la vérité. Oue cela reflète une limitation d'accès ou une méprise sur la fonction pédagogique des procès, il reste que les jugements du TPIY ne suffisent pas à empêcher le déni des crimes et le révisionnisme. Ainsi, un an après le jugement Krstić du 2 août 2001, le rapport commandé par le gouvernement de RS sur le massacre de Srebrenica, était publié le 3 septembre $2002^{20}$. Il chiffrait à 2000 le nombre de Musulmans tués, la plupart au combat, en dénégation manifeste des faits établis par le TPIY.

\section{LE CONSTAT D’UN DÉCALAGE DIFFICILE À INTERPRÉTER}

Aussi, le journaliste Emir Suljagić, correspondant permanent pour Dani à La Haye et lui-même survivant du massacre de Srebrenica dressait en 2003 un constat sévère de l'effet du TPIY sur les criminels et sur les victimes, les uns ne voyant dans le TPIY qu'une institution politique, les autres ayant perdu confiance en lui ${ }^{21}$. Sans effet dissuasif sur des violences ultérieures ou sur les tentatives de révisionnisme, pratiquant une politique d'inculpations et de

19 Celui-ci est constitué de deux personnes pour l'ensemble de la Bosnie, nombre singulièrement peu élevé en regard du personnel employé à La Haye par le tribunal. Les "out reach programs" ne sont d'ailleurs pas financés par le budget du TPIY.

20 Publié par le bureau de liaison du gouvernement de RS avec le TPI.

${ }^{21}$ Conférence prononcée à La Haye au " Grotius Center for international legal studies ", le 20 février 2003. Suljagić (Emir), conférence "The impact of ICTY on the victims and perpetrators of the former Yougoslavia and the importance of justice being done $n$. 
peines peu compréhensible, axé sur la marche de la communauté internationale vers une nouvelle ère de la justice pénale internationale, le TPIY aurait fini par négliger cette partie du monde sur laquelle il a juridiction.

Il rejoint ainsi le constat d'un décalage entre la justice internationale et la demande de justice des intéressés. Selon la journaliste E. Neuffer dans son livre The Key to my Neighbourgh's House. Seeking Justice in Bosnia and Rwanda, H. Weistein ou E. Stover, longtemps médecin légiste pour le TPIY, les victimes, plus soucieuses de la recherche des disparus que de procès, seraient déçues par un tribunal ne répondant pas à leurs attentes ${ }^{22}$.

De fait, même lorsque le tribunal rencontre leurs demandes, sur la question des disparus, les logiques diffèrent. La logique judiciaire est en quête des causes de la mort, alors que les familles recherchent d'abord l'identité des victimes pour enterrer leurs proches. Or, sauf exception, le TPIY ne pratique pas une identification individuelle des corps, qui outrepasserait ses moyens et sa tâche : une identification de groupe par le sexe, l'âge ou les vêtements suffit à établir une qualification en crime de guerre contre des civils, ou en crime contre l'humanité ou génocide contre un groupe donné. Les corps exhumés via les enquêtes du TPIY ne sont généralement pas identifiés dans le cadre des procédures judiciaires.

On est alors tenté de distinguer entre deux sens de la justice, l'une pénale et internationale axée sur l'accusation et les procédures judiciaires, l'autre locale ou nationale, axée sur la recherche des disparus et l'enterrement des morts, ainsi que la reconnaissance des victimes et des témoins. C'est aussi une divergence entre deux genres de recherche de la vérité et de certitude, l'une résultant de l'administration de la preuve, l'autre exprimant une expérience première et douloureuse. Pour les témoins et victimes qui savent par expérience, la vérité est quelque chose que des procès peuvent reconnaître et non établir, rendant difficile la fonction pédagogique d'un tribunal ${ }^{23}$.

Ces analyses, bien mieux informées de la réalité des pays concernés que les précédentes, sont justes pour autant qu'elle s'opposent aux vues des " idéalistes ". Elles ne devraient pas pour autant amener à conclure que, par sa logique même, le TPIY ne peut répondre aux besoins des victimes et des communautés. Le défaut de conclusions de ce type est triple. Premièrement, de telles conclu-

${ }^{22}$ Voir Neuffer (Elizabeth), op.cit., chapitre 9 " Bring me his body " et p. 327 ; Stover (Eric), Shigekane (Rachel), " Les personnes portées disparues au lendemain d'un conflit ", Revue internationale de la CroixRouge, (848), décembre 2002 ; Fletcher (Laurel E.), Weinstein (Harvey M.), art.cit., pp. 634-635.

${ }^{23}$ L. Fletcher et $\mathrm{H}$. Weinstein soulignent très justement que pour les juges bosniaques qu'ils ont interrogés, " la vérité est quelque chose que les procès peuvent reconnaitre mais non quelque chose qui requiert des procédures légales pour être découvert " (Fletcher (Laurel E.), Weinstein (Harvey M.), art.cit., p. 589). J'ai obtenu des réponses analogues dans des entretiens avec des membres ou présidents d'associations de victimes. À la question "Les procès permettent-ils d'établir la vérité ? ", la réponse était "Mais la vérité nous la connaissons ". 
sions n'incluent pas ou peu de perspective de moyen terme, à destination d'une génération qui n'a pas vécu la guerre ${ }^{24}$. Deuxièmement, elles expriment les attentes des idéalistes, déçus de ne pas constater les effets escomptés. Elles montrent tout au plus que la justice n'est pas une condition suffisante de la paix et de la réconciliation et qu'elle ne saurait seule, par simple fiat, combattre les guerres, le révisionnisme, promouvoir la paix et la réconciliation. Elles reproduisent ainsi la naïveté du discours idéaliste croyant à une action directe de la justice dans un vacuum politique et participent ainsi d'une dé-contextualisation que précisément elles reprochent à une justice trop distante ${ }^{25}$.

Or, étant donné le faible nombre d'inculpations et d'arrestations en regard de l'ampleur des crimes, étant donné l'obstruction de nombre d'autorités nationales et locales, il est difficile d'évaluer si la justice et la vérité n'ont pas l'effet supposé ou s'il y a si peu de justice et si peu de connaissance de la vérité que le manque d'effets est seulement proportionnel à la faiblesse des causes. Il est difficile de trancher entre deux interprétations : la justice, même une fois rendue, est sans effets, ou bien, il y a trop peu de justice pour qu'elle ait un effet ${ }^{26}$.

${ }^{24}$ Sur la question des visées de court, moyen et long termes pour de tel tribunaux, voir les analyses très éclairantes et toujours d'actualité de Schklar (Judith), Legalism, Law, Morals and Political Trials, Harvard University Press, $1964,3^{\mathrm{e}}$ partie, notamment p. 164 et sq. Pour la fonction pédagogique des tribunaux à moyen terme, voir aussi Bass (Gary J.), " Milosevic in The Hague n, Foreign Affairs, May/June 2003.

${ }^{25}$ Je renvoie en particulier aux travaux du Human Rights Center de Berkeley mené par L. Fletcher, E. Stover, H. Weinstein et alii notamment à Fletcher (Laurel E.), Weinstein (Harvey M.), " Justice, Accountability, and Social Reconstruction : An Interview Study of Bosnian Judges and Prosecutors n, Berkeley Journal of International Law, 18 (1), 1999 ; Fletcher (Laurel E.), Weinstein (Harvey M.), « Violence and Social Repair " (art.cit.). Ces travaux ont l'immense mérite d'être parmi les premiers à porter sur la réception du TPIY en Bosnie. Et l'on ne peut que souscrire à leur critique des vues des " idéalistes n. On peut toutefois regretter qu'ils ne distinguent pas plus clairement entre la critique de ces vues et les effets réels du TPIY, c'est-à-dire qu'ils n'envisagent le TPIY que du point de vue des discours tenus par ses défenseurs et non de son inscription dans la réalité de la Bosnie d'après-guerre. Tout en prônant une approche holiste du rapport entre justice et reconstruction, leurs analyses ne tiennent guère compte du contexte effectif, notamment dans l'étude sur les juges et procureurs de Bosnie. Il reste à voir si de telles objections seraient toujours valides à l'encontre de l'ouvrage concluant ce projet de recherche (à ce jour non disponible) : Stover (Eric), Weinstein (Harvey), eds., My Neighbor, My Enemy : Justice and Community in the Aftermath of Ethnic Cleansing, Cambridge : Cambridge University Press, 2004.

${ }^{26}$ Ces difficultés d'interprétation se manifestent à propos du récent rapport de RS du 11 juin 2004 reconnaissant le massacre de Srebrenica. On peut voir là le fruit du long travail du TPIY qui rendait de plus en difficile la dénégation du crime face au nombre d'exhumations et aux plaidoyers de culpabilité pour Srebrenica. On peut aussi remarquer que la publication de ce rapport n'est pas seulement consécutive au travail du TPI, mais aussi à de fortes pressions internationales et au remplacement du bureau de liaison avec le TPIY de RS par le Haut Représentant (voir à ce sujet * Bosnian Serb "forced" into atrocity admission ", Tribunal update, (363), 18 juin 2004). Mais une simple comparaison avec des pays aussi pacifiés et démocratiques que la France et l'Allemagne montre que les procès de Nuremberg ou des procès plus récents ne suffisent pas à combattre le révisionnisme sans des dispositifs législatifs contraignants comme la loi Gayssot, aussi discutables soient-ils, et que toute tentative de dissocier reconnaissance de la vérité d'une part, conditions et moyens institutionnels de sa reconnaissance, d'autre part, est, dans de ce genre de contexte, oiseuse. 
Troisièmement, conclure à l'absence d'effets de cette justice reconduirait une opposition dommageable entre " eux " et " nous ", entre le local ou le national et l'international qui ne prend pas en compte l'intrication des points de vue et des interactions ${ }^{27}$.

De telles difficultés de détermination et d'interprétation des critères et des faits pertinents peuvent être partiellement surmontées en croisant différentes approches. D'une part, l'intrication entre le point de vue de la justice internationale et celui des pays et individus concernés se manifeste clairement à propos du critère d'individualisation de la responsabilité ainsi que de la recherche des disparus. D'autre part, la difficulté à trancher entre absence d'effets ou manque de justice peut être éclairée par l'évolution de Prijedor qui a connu le plus grand nombre d'inculpations et de jugements à La Haye. Je concentrerai mes analyses sur le cas de la Bosnie à partir d'une enquête de terrain réalisée en 2002.

\section{L'INDIVIDUALISATION DE LA RESPONSABILITÉ}

En premier lieu, l'idée que l'individualisation de la responsabilité et l'assignation de responsabilités individuelles empêcheront la stigmatisation de groupes entiers est ambiguë. Elle se trouve à l'articulation, d'une part, d'un principe d'individualisation de la responsabilité et de la peine constitutive du droit pénal et, d'autre part, d'une idée libérale qui fait de l'individu le vrai sujet moral et politique, par opposition à la prévalence de la communauté et du groupe. Toutefois, cette idée que l'assignation de responsabilités individuelles empêche la stigmatisation des groupes suscite réserves et objections.

En premier lieu, en raison du nombre limité et sélectif des inculpations et des jugements, une fraction seulement des personnes responsables de violations graves du droit international humanitaire est jugée. Après une guerre ou des crimes de masse, le nombre de responsables et d'exécutants dépasse de loin les capacités de toute institution légale. Et même si tous étaient jugés, ce qui serait techniquement impossible, cela ne résoudrait pas le problème des différents degrés de participation ou de complicité qui apparaissent dans la plupart des témoignages à La Haye décrivant l'implication de voisins, de connaissances ou de " visiteurs ", comme dans les camps de Prijedor. Comme le souligne Bass, les jugements sont nécessairement symboliques : quelques

\footnotetext{
27 Je vise par là toute absolutisation des diffèrences anthropologiques, à l'instar de celle des mentalités. Je me réfère aussi à une conception de l'international et du national comme n'étant pas séparables, voir Lejbowicz (Agnès), Philosophie du droit international, Paris : PUF, 1999. Les différences et les conflits traversent aussi bien le TPIY ou la Bosnie.
} 
criminels sont là pour un groupe plus large de coupables. Ce qui est présenté comme une justice individuelle est un moyen de facto d'exonérer de nombreux coupables. Contre la culpabilité collective, une telle justice suppose « le mythe d'une innocence collective $n^{28}$.

Individualiser la responsabilité se révèle donc une formule ambiguë, source de malentendus. En raison de l'ampleur des crimes et de la sélectivité des inculpations, bien des exactions ne donnent lieu à aucune ou seulement à quelques inculpations. D'où le hiatus entre l'affirmation d'un combat contre l'impunité par la justice internationale et le sentiment d'un triomphe de l'impunité prévalent parmi bien des Bosniaques, qui ne cessent de réclamer justice avec espoir et colère, avec la même véhémence que dans l'immédiat aprèsguerre. C'est que le scandale de l'impunité n'est pas d'abord national ou ethnique. Côté bosnien, que Karadžić et Mladić n'aient toujours pas été arrêtés apparaît à tous comme un scandale, toutefois les Bosniens de Višegrad dénoncent avant tout l'impunité de Milan Lukić, et ceux de Vogošća celle de Poplašen, ceux de Srebrenica celle de Mladić, l'arrestation de nombreux criminels pour Prijedor n'atténuant pas leurs protestations. Le scandale de l'impunité est dénoncé à un niveau individuel, familial ou local qui est celui où sont connus les crimes et les criminels. Supposer que des inculpations d'autres Serbes pour des crimes commis contre d'autres Bosniens dans d'autres municipalités puisse répondre au sentiment d'injustice de ceux qui n'ont pas été victimes de ces criminels-là, ce serait précisément supposer un sens de la culpabilité collective que ces jugements visent à combattre.

Car telle est la difficulté de la visée d'individualisation de la responsabilité par la justice internationale : peut-on apporter ce qui est déjà présent ? Dans les entretiens que j'ai pu réaliser avec des associations de victimes civiles, d'anciens détenus des camps et plus particulièrement de familles de disparus en Bosnie, ou ponctuellement à Belgrade, mes interlocuteurs identifiaient individuellement ceux qu'ils dénoncent comme des criminels par leur nom propre, leur photo, leur fonction ou leur lien avec les témoins et liaient étroitement la recherche des disparus avec la désignation des responsables de la disparition. Au niveau personnel, local ou régional où les crimes sont les plus directement dénoncés, l'individualisation est opérée sur la base de l'inscription des accusés dans un réseau local de pouvoir et d'institutions (chef de police, de tel parti politique, ancien maire) ou de relations personnelles de voisinage ou de travail. Selon la responsable locale de la commission fédérale des personnes disparues de Mostar, à la recherche de ses enfants, " si je parle en général des Serbes de Nevesinje qui ont commis tous ces 
crimes, c'est comme si je ne disais rien, mais si je nomme un tel, un tel et un tel, et les autres, là je dis quelque chose. Derrière chaque crime, il y a un nom et un prénom ${ }^{29}$.

Une telle individualisation sur le terrain engage à interroger le discours de la justice pénale internationale. Y a-t-il adéquation de points de vue entre cette justice et la demande de justice des victimes, tout au moins en Bosnie ? Il s'agirait plutôt d'un croisement que d'une adéquation de perspectives.

Stricto sensu, la justice internationale n'individualise pas les responsabilités : elle juge des individus dont elle détermine et hiérarchise la responsabilité pénale selon des procédures plus qu'elle n'assigne des responsabilités individuelles dans un groupe donné (les Serbes de Srebrenica par exemple). En revanche, la plupart des victimes individualisent mais ne hiérarchisent guère les responsabilités, pas plus qu'elles ne jugent pertinente la distinction entre responsabilité criminelle et responsabilité politique. D'où une insatisfaction envers une justice internationale qui en visant les plus haut responsables, tout au moins dans sa stratégie d'achèvement actuelle, laisse de côté les simples exécutants ou les responsables locaux de moindre envergure, les plus aisément identifiés par les victimes et les témoins.

Enfin, il est de jure et de facto douteux que l'assignation de responsabilité individuelle suffise à éliminer l'idée de responsabilité ou de culpabilité collective. D'une part, elle suppose a contrario une innocence collective qui ne correspond guère à l'expérience des survivants. D'autre part, elle suppose que des procédures d'individualisation s'opposent au collectif et le déconstruisent. Or désignation individuelle et identification de soi-même et des autres par l'appartenance à un groupe ne sont pas exclusifs aux yeux de nombre de Bosniaques, soit que la somme d'individus identifiés finisse par former un collectif, soit que l'individualisation passe par le rattachement à une communauté.

$\mathrm{Au}$ demeurant, dans la logique du droit international, l'individualisation de la responsabilité pénale ne se distingue pas tant de la responsabilité collective des groupes que de celle des États. L'incrimination des États et des individus est alors compatible ${ }^{30}$. Le TPIY n'est pas la seule juridiction à être appelée à se prononcer sur les crimes commis en ex-Yougoslavie. En 1993, la B.-H. a déposé une plainte contre la Yougoslavie pour génocide, plus tard suivie par la Croatie, plaintes sur lesquelles la Cour Internationale de Justice de La Haye

\footnotetext{
29 Ces propos rapportés dans le film de Subašič (Sabina), Gardel (Fabrice), Viol. Une arme de guerre, 2002 , sont très représentatifs de ceux entendus dans nombre d'entretiens. Ils se poursuivent ainsi : « je sais bien qu'ils étaient dirigés par Slobodan Milošević, mais tout de même, Dieu a donné à chacun de nous la raison pour qu'il puisse décider de tuer ou ne pas tuer, d'être ou ne pas être un humain digne de ce nom $n$.

${ }^{30}$ De fait, le jugement de Guillaume II a été envisagé en sus de la condamnation de l'Allemagne à payer des réparations au traité de Versailles.
} 
s'est déclarée compétente. Un jeu complexe sur l'individuel et le collectif se joue alors entre le discours des partis politiques et des représentants officiels : le SDA voit dans les jugements de La Haye la confirmation de la nature criminelle de la RS et un appui à la plainte contre la Yougoslavie, les représentants de RS ne voient les jugements ou les reconnaissances de culpabilité que comme ceux d'individus, sans conséquence institutionnelle ${ }^{31}$, alors que le discours de la justice internationale attend des jugements et des reconnaissances de culpabilité individuelles un effet collectif en termes de réconciliation ${ }^{32}$.

Il serait donc inadéquat d'opposer une logique d'individualisation qui serait celle d'une justice internationale "libérale " à une logique collective, ethnique ou nationaliste. Une telle opposition, douteuse pour l'individualisation des responsables, le serait plus encore pour l'individualisation des victimes. De fait, concernant le traitement des morts, la justice internationale reste dans une logique de groupe, la catégorisation des victimes selon le sexe, l'âge ou le groupe national suffisant à une inculpation pour crime de guerre, crime contre l'humanité ou génocide. La recherche de l'identification individuelle des victimes est celle des familles ou des communautés, ainsi que des organisations humanitaires qui les soutiennent.

\section{LA RECHERCHE DES DISPARUS}

Quoique la visée d'individualisation des victimes soit première pour les familles, on ne peut séparer justice internationale et recherche des disparus. Du point de vue même des familles, la recherche des causes et des responsables de la disparition n'est pas d'une moindre importance que la recherche des disparus. La demande d'identification des corps et celle de jugement des coupables ne sont pas séparables : la découverte des corps contribue à l'identification des responsables et inversement les familles espèrent retrouver les corps grâce aux procès.

Cette interaction entre le local, le national et l'international, ainsi que le rôle et les limites d'une justice internationale en fonction des médiations qu'elle requiert ou représente, s'éclairent par la confrontation entre le cas des disparus bosniaques de Hadžići, dans la banlieue de Sarajevo, ceux de Srebrenica et celle des disparus serbes de Krajina croate. À Hadžići, où l'association des disparus recherche 300 corps, les charniers ou fosses sont directe-

${ }^{31} \mathrm{Ce}$ jeu sur le jugement des individus et ses conséquences individuelles ou collectives est particulièrement clair dans les déclarations de Mladen Ivanić (premier ministre de RS) et de Sulejman Tihić (membre bosnien de la présidence de B.-H.) à propos du jugement Plavšić. Voir par exemple Oslobodjenje du $04 / 10 / 02$ et du $28 / 02 / 03$.

$3^{2}$ Voir à ce propos le texte du jugement de Plavšić. 
ment accessibles par les familles et la commission fédérale des personnes disparues, instance nationale qui y a déjà pratiqué de nombreuses exhumations. Cependant, bien des corps ne sont pas localisés, et ne pourraient l'être que par les indications des responsables des disparitions. Ceux-ci sont hors d'atteinte des autorités de la Fédération et ne pourraient être jugés qu'à La Haye suite à une inculpation du TPI, inexistante à ce jour, ou dans des procès locaux, avec l'aval du TPIY. À Srebrenica, en RS, à la fois les arrestations et l'accès au charnier sont dépendants des inculpations et des enquêtes du TPIY ; les exhumations et les identifications s'opèrent par une collaboration entre TPIY, commission fédérale et ICMP (International Commission for Missing Persons). Pour les disparus serbes de Krajina croate, en l'absence de coopération des autorités croates, les inculpations mais aussi les exhumations sont entièrement dépendantes des inculpations du TPIY pour l'opération " Tempête ". Pour l'association des familles de disparus serbes en Croatie, située à Belgrade, le TPIY est la condition sine qua non d'accès aux corps de leurs proches 33 .

Dès lors, la contribution de la justice internationale à la justice nationale et à la demande de justice des intéressés ne peut être évaluée isolément, mais seulement dans sa fonction de médiation en rapport avec des institutions locales et nationales qu'elle supplée, complète ou tente d'infléchir. Condition non nécessaire pour l'accès aux charniers de Hadžići, elle le reste pour le jugement des criminels présumés faute de coopération judiciaire entre Fédération et RS, soit directement par des procès à La Haye, soit indirectement par l'aval donné à des procès locaux ${ }^{34}$. Condition nécessaire pour l'accès aux charniers et pour l'inculpation des criminels présumés de l'opération " Tempête ", elle est aussi restée non suffisante aussi longtemps que les autorités croates refusaient ou refusent encore l'arrestation de généraux inculpés ou l'accès à d'autres charniers. Condition nécessaire pour les inculpations et les exhumations à Srebrenica, le TPIY est néanmoins jugé insuffisant face à l'ampleur du crime pour la localisation 35 et l'exhumation des charniers et ne contribue qu'indirectement à une tâche d'identification des morts essentiellement assurée, comme ailleurs, par les commissions nationales, les associations locales, le CICR et le ICMP qui a mis en place un programme d'identification par ADN.

33 Il convient de préciser que l'entretien réalisé à Belgrade avec l'association des familles de disparus serbes de Croatie a eu lieu en juin 2003 avant le départ à La Haye de certains des généraux croates inculpés pour l'opération " Tempête ".

${ }^{34}$ L'entretien réalisé avec l'association des familles de disparus de Hadžići a eu lieu en juillet 2002. Depuis le procès local de Vlastimir Pušara a débuté à Sarajevo.

35 Notons que le très récent rapport de RS sur Srebrenica a révélé 32 localisations de charniers encore inconnues. 


\section{ARRESTATIONS ET RETOURS À PRIJEDOR}

Enfin, la situation actuelle de Prijedor permet de lever partiellement la difficulté à déterminer s'il y a trop peu d'effets de la justice ou trop peu de justice pour qu'elle ait un effet. À ce sujet, je me réfère à la fois à des entretiens réalisés en personne, aux rapports des organisations internationales, sur le retour des réfugiés notamment, à des articles de la presse bosniaque, et au livre récent de deux journalistes, Isabelle Wesseling et Arnaud Vaulerin, Bosnie, la Mémoire à vif, centré sur Prijedor aujourd'hui ${ }^{36}$. Pour les camps d'Omarska, Trnopolje, Keraterm et le massacre du mont Vlašić, Prijedor a concentré le plus grand nombre d'inculpations et d'arrestations au TPIY. Tadić est arrêté dès 1994 et Drljača, l'ancien chef de la police, dès 1997. Toutes les personnes officiellement inculpées (19) sont à ce jour soit décédées soit jugées ou en cours de jugement. Outre ces inculpations spécifiques, les crimes de Prijedor apparaissent aussi dans les procès Brdjanin, Plavšić, Krajišnik, Milošević, etc. Les inculpations ont visé non seulement les principaux responsables locaux (maire, chef de la police, etc.) mais aussi des figures symboliques associées aux différents types d'exactions : directeur de camps comme Meakić ou "visiteurs " de camps comme Žigić, responsable, publiquement dénoncé par les Bosniens, du massacre du Mont Vlašić comme Mrdja.

Constate-t-on une poussée nationaliste dans cette région ? Non. À la domination du SDS pendant la guerre a succédé l'élection d'une mairesse SNDS, Nada Sevo, en 2000, qui a obtenu la majorité par une alliance avec les Bosniens de retour. Constate-t-on un effet pédagogique du tribunal ? Guère plus, la presse locale ne couvre pas les procès, aucune plaque commémorative officielle ne mentionne les victimes des camps d'Omarska, Trnopolje et Keraterm. Les survivants de ces camps perçoivent comme une dénégation et une célébration du crime l'érection d'un monument aux soldats serbes morts pour la RS devant l'ancien camp de Trnopolje, redevenu une école dont la fête coïncide maintenant avec le jour de l'ouverture du camp. À l'instar du discours officiel des représentants de la RS, l'individualisation de la responsabilité est invoquée par un prêtre orthodoxe pour limiter les responsabilités à des individualités, sans rapport avec la communauté serbe ${ }^{37}$. L'instauration de la paix ? Assurément la région n'est plus en conflit et relativement sûre pour les Bosniens de retour. Mais c'est moins dû à une action à distance du TPIY qu'à

\footnotetext{
${ }^{36}$ Wesseling (Isabelle), Vaulerin (Arnaud), Bosnie, la Mémoire à vif, Paris : Buchet Chastel, 2003. Cet ouvrage est, à ce jour, l'un des plus justes dans l'évaluation des rapports du TPIY et de la Bosnie. Pour la relation entre les jugements de La Haye et la situation de Prijedor après-guerre, voir aussi l'ouvrage Neuffer (Elizabeth), op.cit.
}

${ }^{37}$ Cité dans Wesseling (Isabelle), Vaulerin (Arnaud), op.cit., p. 92. 
l'efficacité de la S-FOR qui a aussi permis l'arrestation rapide des inculpés, à la différence de la région de Foča. C'est toutefois une pacification relative. Prijedor a connu en 2002, avec Mostar, le plus grand nombre d'incidents violents en Bosnie, contre des bâtiments religieux ou en relation avec des événements sportifs, mais non avec le calendrier de La Haye. Et la découverte en septembre 2003 par la S-FOR de plus de 4 tonnes d'armes et de munitions à Prijedor peut laisser sceptique sur la solidité de cette pacification.

Le TPIY est-il donc sans effets ? On peut constater un fait marquant à Prijedor : la ville concentre à la fois le plus grand nombre de jugements du TPIY et un grand nombre de retours de Bosniens en RS dans l'un des lieux de plus grande violence pendant la guerre. Parmi les diverses motivations du retour, les réfugiés invoquent l'arrestation des criminels de guerre qui les avaient persécutés dans les camps et continuaient d'empêcher leur retour, comme l'ancien chef de la police Drljača. Un tel retour est-il donc un critère de réconciliation et de contribution de la justice internationale à une justice reconstructive du lien social ? Les deux communautés tendent à vivre dans des villages et mondes séparés et les " returnees " créent des institutions parallèles. Il s'agit plutôt de coexistence pacifique que d'un vivre ensemble. Il reste qu'un tel retour de ceux qui avaient été chassés est quasi inexistant là où les inculpations et arrestations sont minimes ou très tardives comme à Srebrenica ou en Herzégovine. Une possible corrélation entre arrestations et jugements, d'une part, retour des victimes de violence, d'autre part, ne peut toutefois être systématisée, mais plutôt être étudiée au cas par cas, de nombreux jugements pour Foča n'ayant pas donné lieu à un tel phénomène de retour.

Si l'on combine donc une analyse des discours et des pratiques d'individualisation des différents acteurs locaux, nationaux et internationaux pour un procès donné, une approche transversale et comparative entre différentes régions sur une question donnée, comme la recherche des disparus, une approche géographique où interagissent plusieurs facteurs, comme à Prijedor, l'idée d'un découplage entre justice internationale et sens de la justice national et local n'est guère opératoire. La place de la justice internationale dans un processus de sortie de violence n'a de sens que dans son contexte et en relation avec ses alternatives, impunité totale, exécutions sommaires ou procès locaux.

Or, la primauté accordée au TPIY a pour effet que des procès locaux pour crime de guerre ne peuvent avoir lieu en Bosnie sans obtenir l'aval du TPIY, via l'accord de Rome et les " Rules of the Road ". Celles-ci visent à éviter une politisation des inculpations ou des procès menés à partir de preuves inexistantes ou douteuses, et sans garantie des droits de la défense, comme le procès Damjanović, condamné à mort à Sarajevo en mars 1993 pour le meurtre de deux frères toujours vivants. Les procès locaux pour crimes de guerre ayant eu lieu en B.-H. offrent peu de garantie de protection des témoins souvent soumis 
à des intimidations, quand ils ne sont pas assassinés, comme cela s'est produit plusieurs fois en Croatie. La difficulté n'est pas seulement celle d'un manque de moyens mais aussi celle du manque de respect de l'anonymat par les instances judiciaires et extra-judiciaires. Le 29 mars 2002, le contenu d'enveloppes hautement confidentielles allant de La Haye vers le ministère de la justice de B.-H. via la procédure des "Rules of the Road ", s'est retrouvé publié dans le journal Jutarnje Novine avec le nom des criminels présumés et des témoins protégés.

Aussi, dans divers entretiens, les témoins ou potentiels témoins, quelles que soient leurs réticences, leurs insatisfactions envers le TPIY et leur crainte d'aller témoigner, manifestent une confiance (ou, tout au moins, ne manifestent pas une défiance) envers cette institution qu'ils n'éprouvent pas envers les cours locales qu'ils jugent incapables d'assurer leur protection ou de mener à bien des procès. Le respect et la protection des témoins (dont on crédite davantage la justice internationale) apparaissent alors comme une norme morale qui établit une distance envers les institutions locales.

\section{CONCLUSION}

Revenons en conclusion à la question initialement posée des critères d'évaluation de la justice internationale dans l'après-guerre. Aucun des critères proposés pour s'opposer à cette justice ou la promouvoir n'est directement opératoire ni non plus impertinent. Une première difficulté est celle de la détermination des critères empiriques de ces principes de justice. À quelle aune par exemple mesurer un concept aussi flou que la réconciliation : à la défaite électorale des partis nationalistes ? À l'échange d'excuses et à la demande de pardon? Au nombre de retours ? Une deuxième difficulté est que pour tout effet empirique retenu, il sera lui-même sujet à une pluralité d'interprétation où le TPIY ne sera qu'un facteur parmi d'autres, que ce soit de choix électoral ou de choix de retour. Aussi le calendrier des activités du TPIY ne rentre-t-il guère en corrélation systématique ni avec les incidents violents, ni avec les résultats électoraux, ni avec les diverses phases de retour. La complexité des situations empiriques, comme celle de Prijedor ou de la recherche des disparus, peut amener à réviser ces critères jusqu'à leur dilution, elle peut aussi amener à les reformuler, au cas par cas, dans des termes à la fois empiriquement observables et pourvus de sens pour les Bosniaques. Le concept de réconciliation peut ainsi être confronté aux diverses formes de retour, aussi problématiques soient-elles ${ }^{38}$.

$3^{8}$ À condition de ne pas prendre le retour comme un critère de justice allant de soi et le droit au retour comme un absolu. 
Évaluer les effets de cette justice ne peut se faire par la projection sur une situation spécifique d'après-guerre de principes émanant d'une théorisation $a$ priori de la justice internationale ne tenant pas compte de la dimension transitoire et révisable de toute analyse, de l'évolution des pays concernés, de l'articulation entre institutions internationales et nationales et demande de justice locale, que ce soit celle de procès et de peines équitables, de respect des témoins ou d'accès aux disparus.

C'est aussi l'évaluation philosophique des critères de la justice internationale qui se trouve posée. Selon John Rawls dans The Law of Peoples, la justice doit contribuer à la justice, ce qui n'est pas une tautologie, ni une forme remaniée d'un fiat justitia, pereat mundus. C'est l'idée que la justice internationale ne doit viser ni sa propre perpétuation, ni le simple accroissement du bien-être et de la richesse mais la préservation ou l'établissement d'institutions justes pour les communautés concernées. Selon ce critère, c'est la contribution à la justice d'institutions nationales et locales et la capacité du TPIY a énoncer des normes publiques de justice (par exemple, de respect des témoins), qui permettront de le qualifier de juste plutôt que des avancées du droit international. Cette conception s'oppose également à une vision plus radicalement cosmopolitique qui fait de l'individu et non des institutions nationales la fin première de toute justice internationale.

Pour juger d'une telle articulation entre l'individuel et l'institutionnel, considérons les trois cas suivants. Pour les inculpés à La Haye, la justice internationale garantit directement des droits. Inversement, dans les procès locaux, elle ne donne directement aucun droit aux accusés ou aux témoins, mais seulement par l'intermédiaire d'un contrôle minimal sur des institutions locales. Pour les familles de disparus serbes en Croatie, elle est une médiation entre des individus et l'exercice de certains droits ou de certaines aspirations, sans garantie institutionnelle et sans guère de collaboration avec des institutions nationales.

La contribution de la justice internationale à la justice nationale ou locale peut donc être directe ou médiate. Elle peut se formuler en termes institutionnels (par la préservation ou la réforme des institutions locales), ou individuels (en termes de droits ou de capacités des individus concernés). Ces institutions ne sont pas seulement juridiques mais également sociales, comme l'enterrement des morts, et la justice pour les individus ne relève pas seulement de la garantie de droits mais aussi de l'exercice de certaines capacités ou libertés, comme celle de savoir ce qui s'est passé.

Quel que soit le jugement que l'on pourra porter sur le TPIY, sa contribution à la sortie de violence et à une justice d'après-guerre, devrait être évaluée par l'articulation de ces critères dans leur dimension à la fois institutionnelle et individuelle. 